\title{
Managing the green proteomes for the next decade of plant research
}

\author{
Andrew W. Carroll ${ }^{1}$, Hiren J. Joshi ${ }^{2}$ and Joshua L. Heazlewood ${ }^{1 *}$ \\ ' Department of Cellular and Molecular Medicine, Copenhagen Center for Glycomics, University of Copenhagen, Copenhagen, Denmark \\ 2 Physical Biosciences Division and Joint BioEnergy Institute, Lawrence Berkeley National Laboratory, Berkeley, CA, USA \\ *Correspondence: jlheazlewood@lbl.gov \\ Edited by: \\ Richard A. Jorgensen, Carnegie Institution for Science, USA
}

Keywords: proteomics, informatics, database, phosphorylation, proteogenomic, subcellular

For the past decade the field of proteomics has transitioned from a highly specialized research area into a conventional technique widely employed by plant biologists. This approach now encompasses basic protein identification to advanced comparative studies. The result has been an abundance of proteomics data, often not readily available to the research community (Heazlewood, 2011). This has resulted in the creation of numerous proteomic resources which are often referred to as boutique databases. Generally, these sites exist outside the traditional community driven centralized repositories. While the geographic location of web-based resources is somewhat inconsequential, it can highlight active regions of plant proteomic-based research. The intention of this Research Topic on Plant Proteomic Resources is to collect articles focusing on these resources and provide an overview of current online plant proteomic portals.

Plant proteomic resources are often integrative and comprise collections of diverse 'omics information to support the proteomic data. A good example of this is the GabiPD portal (Usadel et al., 2012), the website is a gateway for the German plant community to codify and unite various research programs at one site. More focused resources such as pep2pro was constructed to support large-scale proteomic surveys in the model plant Arabidopsis (Hirsch-Hoffmann et al., 2012). The pep2pro repository employs a unique workflow to match spectral data directly against the Arabidopsis genome. Although techniques for arraying proteins by $2-D E$ have been employed for decades, the GelMap portal links proteomic-based identifications with gel electrophoresis maps (Senkler and Braun, 2012). The GelMap resource provides annotated two-dimensional arrays of proteins from a range of sample types.

The application of proteomics to characterize organelles were some of the first large-scale surveys in plants. The AT_CHLORO database represents the most extensive analysis of the chloroplast from the model plant Arabidopsis (Bruley et al., 2012). This resource provides a compendium of proteins identified in the chloroplast and contains information on its subcompartments e.g., thylakoid. Organelle proteome databases such as AT_CHLORO comprised many of the early online plant proteomic databases including the mitochondrion (Heazlewood and Millar, 2005) and the peroxisome (Reumann et al., 2004). The latter was recently used to develop a new resource, PredPlantPTS1, which predicts whether a protein will localize to the peroxisome (Reumann et al., 2012). The SUBcellular Arabidopsis database (SUBA) contains data from most subcellular proteomic surveys in Arabidopsis (Tanz et al., 2013). A similarly focused resource, the Plant Protein DataBase (PPDB) also deals with subcellular proteomics but also encompasses other plant species (Sun et al., 2009). Although the latter two resources are not part of this collection, data housed by these repositories are available through the MASCP Gator, a portal designed to aggregate Arabidopsis proteomic data for the community. The MASCP Gator interface was developed to provide a mechanism for proteomic data visualization from multiple data sources (Mann et al., 2013).

The model plant Arabidopsis dominates the plant proteomic resource landscape, but as genomic information in other plant species becomes available, databases for other species have been established. The rice RNA-binding protein resource provides a curated collection of over 250 experimentally identified RNA interacting proteins from rice (Doroshenk et al., 2012), providing functional annotation, expression, and phylogenetic relationships. Large-scale developmental and organ specific analyses of the rice proteome has now, also been conducted. These data are available through the rice proteogenomics database (OryzaPGDB) which provides a visual relationship between the genome and the identified proteome (Helmy et al., 2012). The Soybean Proteome Database (SPD) initially focused on curating proteins that were responsive to flooding (Ohyanagi et al., 2012), but it now includes a host of 2-DE arrayed organelle proteomes, expression information and information on other stress induced proteins from this important leguminous crop.

Seed development represents a major agricultural focus for plant researchers and as such, this developmental process has been extensively targeted by proteomic surveys. The seed proteome web portal provides an extensive collection of data, including quantitative information on proteins involved in seed development (Galland et al., 2012). As is the case with the Seeds of Chernobyl resource, which highlights a different aspect of seed development in plants, namely cataloging the effects of ionizing radiation on seed maturation and development (Klubicova et al., 2012).

Post-translational modifications (PTMs) often represent the functional state of a protein and are a significant objective for many proteomic studies. A number of resources have been developed to interact with these phosphoproteomic datasets. Initial phosphoproteomic surveys involved Arabidopsis and one of the first phosphorylation-based databases created in any species was PhosPhAt (Arsova and Schulze, 2012). The resource contains many thousands of experimentally identified sites available in the literature. The expansion of phosphoproteomic surveys outside Arabidopsis has resulted in the creation of two further resources, 
the $\mathrm{P}^{3} \mathrm{DB}$ database houses tens of thousands of phosphopeptides from six plant species. The collection of such an array of data by $\mathrm{P}^{3} \mathrm{DB}$ led to the development of Musite, a utility that predicts phosphorylation sites in plant proteins (Yao et al., 2012). Lastly, the Medicago PhosphoProtein Database houses data from a recent large-scale phosphoproteomic analysis of this model legume plant (Rose et al., 2012).

The proteomics community has created an array of online tools that can be used to support various technical approaches in mass spectrometry. The MRMaid utility was designed to facilitate the selection of peptides for targeted proteomic analyses (Fan et al., 2012). The tool leverages plant spectral information housed in the PRIDE repository (Vizcaino et al., 2013) to assist in the selection of protein specific peptides for multiple reaction monitoring (MRM) of plant samples. In a similar vein, the ProMEX resource enables newly collected tandem mass spectrometry data to be queried against previously matched experimental spectra (Wienkoop et al., 2012). Spectral matching process provides real world context as tandem mass spectra generally do not produce evenly distributed fragment ions.

The range of proteome resources highlighted in this Research Topic reflect the diversity of proteomic-based applications in plant sciences. The principle objective for many these research groups has been focused on cataloguing or collecting data in an effort to capture information. Indeed, the creation of many data portals likely reflects an attempt to make sense of one's own data. This collection highlights the diversity and range of plant proteomic resources and utilities available to the plant research community.

\section{ACKNOWLEDGMENTS}

This work conducted by the Joint BioEnergy Institute was supported by the Office of Science, Office of Biological and Environmental Research, of the U.S. Department of Energy under Contract No. DE-AC02-05CH11231.

\section{REFERENCES}

Arsova, B., and Schulze, W. X. (2012). Current status of the plant phosphorylation site database PhosPhAt and its use as a resource for molecular plant physiology. Front. Plant Sci. 3:132. doi: 10.3389/fpls.2012.00132

Bruley, C., Dupierris, V., Salvi, D., Rolland, N., and Ferro, M. (2012). AT_CHLORO: A chloroplast protein database dedicated to sub-plastidial localization. Front. Plant Sci. 3:205. doi: 10.3389/fpls.2012.00205

Doroshenk, K. A., Crofts, A. J., Morris, R. T., Wyrick, J. J., and Okita, T. W. (2012). RiceRBP: a Resource for Experimentally Identified RNA Binding Proteins in Oryza sativa. Front. Plant Sci. 3:90. doi: 10.3389/fpls.2012.00090

Fan, J., Mohareb, F., Jones, A. M., and Bessant, C. (2012). MRMaid: The SRM assay design tool for Arabidopsis and other species. Front. Plant Sci. 3:164. doi: 10.3389/fpls.2012.00164

Galland, M., Job, D., and Rajjou, L. (2012). The seed proteome web portal. Front. Plant Sci. 3:98. doi: 10.3389/fpls.2012.00098

Heazlewood, J. L. (2011). The green proteome: challenges in plant proteomics. Front. Plant Sci. 2:6. doi: 10.3389/fpls.2011.00006

Heazlewood, J. L., and Millar, A. H. (2005). AMPDB: the Arabidopsis mitochondrial protein database. Nucleic Acids Res. 33, D605-D610. doi: 10.1093/nar/gki048
Helmy, M., Sugiyama, N., Tomita, M., and Ishihama, Y. (2012). The rice proteogenomics database OryzaPG-DB: development, expansion, and new features. Front. Plant Sci. 3:65. doi: 10.3389/fpls.2012.00065

Hirsch-Hoffmann, M., Gruissem, W., and Baerenfaller, K. (2012). pep2pro: the high-throughput proteomics data processing, analysis, and visualization tool. Front. Plant Sci. 3:123. doi: 10.3389/fpls.2012.00123

Klubicova, K., Vesel, M., Rashydov, N. M., and Hajduch, M. (2012). Seeds in Chernobyl: the database on proteome response on radioactive environment. Front. Plant Sci. 3:231. doi: 10.3389/fpls.2012.00231

Mann, G. W., Calley, P. C., Joshi, H. J., and Heazlewood, J. L. (2013). MASCP Gator: an overview of the Arabidopsis proteomic aggregation portal. Front. Plant Sci. 4:411. doi: 10.3389/fpls.2013.00411

Ohyanagi, H., Sakata, K., and Komatsu, S. (2012). Soybean Proteome Database 2012: update on the comprehensive data repository for soybean proteomics. Front. Plant Sci. 3:110. doi: 10.3389/fpls.2012.00110

Reumann, S., Buchwald, D., and Lingner, T. (2012). PredPlantPTS1: A web server for the prediction of plant peroxisomal proteins. Front. Plant Sci. 3:194. doi: 10.3389/fpls.2012.00194

Reumann, S., Ma, C., Lemke, S., and Babujee, L. (2004). AraPerox. A database of putative Arabidopsis proteins from plant peroxisomes. Plant Physiol. 136, 2587-2608. doi: 10.1104/pp.104.043695

Rose, C. M., Venkateshwaran, M., Grimsrud, P. A., Westphall, M. S., Sussman, M. R., Coon, J. J., et al. (2012). Medicago phosphoprotein database: a repository for Medicago truncatula phosphoprotein data. Front. Plant Sci. 3:122. doi: $10.3389 /$ fpls.2012.00122

Senkler, M., and Braun, H. P. (2012). Functional annotation of 2D protein maps: the GelMap portal. Front. Plant Sci. 3:87. doi: 10.3389/fpls.2012. 00087

Sun, Q., Zybailov, B., Majeran, W., Friso, G., Olinares, P. D., and Van Wijk, K. J. (2009). PPDB, The plant proteomics database at Cornell. Nucleic Acids Res. 37, D969-D974. doi: 10.1093/nar/gkn654

Tanz, S. K., Castleden, I., Hooper, C. M., Vacher, M., Small, I., and Millar, H. A. (2013). SUBA3: a database for integrating experimentation and prediction to define the SUBcellular location of proteins in Arabidopsis. Nucleic Acids Res. 41, D1185-D1191. doi: 10.1093/nar/gks1151

Usadel, B., Schwacke, R., Nagel, A., and Kersten, B. (2012). GabiPD - the GABI primary database integrates plant proteomic data with gene-centric information. Front. Plant Sci. 3:154. doi: 10.3389/fpls.2012.00154

Vizcaino, J. A., Cote, R. G., Csordas, A., Dianes, J. A., Fabregat, A., Foster, J. M., et al. (2013). The Proteomics Identifications (PRIDE) database and associated tools: status in 2013. Nucleic Acids Res. 41, D1063-D1069. doi: 10.1093/nar/ gks1262

Wienkoop, S., Staudinger, C., Hoehenwarter, W., Weckwerth, W., and Egelhofer, V. (2012). ProMEX - a mass spectral reference database for plant proteomics. Front. Plant Sci. 3:125. doi: 10.3389/fpls.2012. 00125

Yao, Q., Gao, J., Bollinger, C., Thelen, J. J., and Xu, D. (2012). Predicting and analyzing protein phosphorylation sites in plants using Musite. Front. Plant Sci. 3:186. doi: $10.3389 /$ fpls.2012.00186

Received: 20 November 2013; accepted: 22 November 2013; published online: 16 December 2013.

Citation: Carroll AW, Joshi HJ and Heazlewood JL (2013) Managing the green proteomes for the next decade of plant research. Front. Plant Sci. 4:501. doi: 10.3389/fpls. 2013.00501

This article was submitted to Plant Proteomics, a section of the journal Frontiers in Plant Science.

Copyright () 2013 Carroll, Joshi and Heazlewood. This is an open-access article distributed under the terms of the Creative Commons Attribution License (CC BY). The use, distribution or reproduction in other forums is permitted, provided the original author(s) or licensor are credited and that the original publication in this journal is cited, in accordance with accepted academic practice. No use, distribution or reproduction is permitted which does not comply with these terms. 